\title{
Questions and Answers in EBD Volume 6
}

\author{
Derek Richards \\ Editor, Evidence-based Dentistry
}

Evidence-Based Dentistry (2005) 6, 104-107. doi:10.1038/sj.ebd.6400363

As in previous years we are high-lighting the questions and answers addressed by summaries in EBD. ${ }^{1-3}$ Evidence levels ${ }^{4}$ are only given for those papers achieving level $3 \mathrm{~A}$ and above.
1. Lawrence A, Richards D. Questions answered in EBD Volumes 1 and 2. Evid Based Dent 2002; 3:27-29.

2. Richards D. Questions and answers in EBD Volumes 3 and 4. Evid Based Dent 2003; 4: 94-97.
3. Richards D. Questions and answers and guidelines in EBD Volume 5. Evid Based Dent 2004; 4: 110-112.

4. Richards D. Not all evidence is created equal - so what is good evidence? Evid Based Dent 2003; 4:17-18.

\begin{tabular}{l} 
Topic Question \\
\hline Dental anxiety \\
Do behavioural interventions contribute to anxiety \\
reduction and result in significantly improved dental \\
attendance in adults? \\
Dental caries \\
Does drinking fluoridated milk help prevent dental \\
caries in children?
\end{tabular}
caries in children?

How effective is fluoride toothpaste in preventing caries?

Can dietary measures assist in the prevention of dental caries?

Is ozone effective in arresting or reversing the progression of dental caries?

In pre-school children at high risk of developing dental caries can glass ionomer fissure sealant placed on primary molars decrease the incidence of dental caries?

Are systemic antibiotics effective in providing pain relief in people who have irreversible pulpitis?

Are systemic antibiotics effective in providing pain relief in people who have irreversible pulpitis?

How good is DIAGNOdent (DD) at detecting caries?

\section{Dental implants}

Is there any difference in implant survival and complications between implants placed in augmented maxillary sinus and non-augmented posterior maxilla?
Behavioural interventions have a positive effect on dental fear, and should, therefore, be incorporated in any treatment of highly fearful patients.

The two studies that were of sufficient quality to be included in this review suggest that fluoridated milk was beneficial to schoolchildren by helping prevent caries in the permanent dentition. However, there is a dearth of studies providing high-quality evidence of the effects of fluoridated milk.

This review reinforces the importance of daily toothbrushing with fluoridated toothpastes for preventing dental caries, although long-term studies in age groups other than children and adolescents are still lacking. The review clearly demonstrates the need for welldesigned randomised clinical studies, with adequate control groups and high compliance, looking at the effect of dietary measures on dental caries.

There is a need for more evidence of appropriate rigour before the clinical implications of this emerging technology can be evaluated.

The available evidence does not support the use of GI as a sealant in primary molars.

There is no evidence to support the use of prophylactic antibiotics in the management of localised endodontic disease in healthy patients.

There is no evidence to support the use of prophylactic antibiotics in the management of localised endodontic disease in healthy patients; adverse drug reactions, while under-reported, are very common and can be life threatening.

In the limited studies available, DD demonstrated greater sensitivity but poorer specificity than visual caries diagnosis. Combined with the fact that little in vivo evidence is available for DD performance, the greater number of false-positive diagnoses suggest it should not be relied on as a clinician's primary diagnostic method.

Implant survival appears to show greater variability in grafted sinuses than in the posterior maxilla. However, well-designed prospective studies with larger patient numbers and control of confounding factors are urgently needed.

Evidence Page

level no.

2B

$1 \mathrm{~A}$

$1 \mathrm{~A}$

$1 \mathrm{~A}$

$1 B$

1B

1B

1B

$3 A$ 64-65

2B 
Endodontics

What is the optimal obturation length of a root canal to achieve the best prognosis?

\section{Evidence-based practice}

Is evidence-based medicine teaching more effective when integrated into routine practice or when taught in stand-alone workshops?

Which is the best critical appraisal tool to evaluate the quality of allied health research?

\section{Oral cancer}

What is the global prevalence of leukoplakia?

Do bidi smokers have an increased risk for oral cancer?

Is there a dose-risk relationship between alcohol and disease?

\section{Oral health promotion}

Is a triclosan/copolymer and fluoride dentifrice more effective at improving plaque control and gingival health than a fluoride dentifrice or no intervention?

Does oral health promotion improve oral hygiene and gingival health?

\section{Oral medicine}

What is the relative efficacy of single-dose oral analgesics after third-molar extraction?

What treatments are effective for treating oral leukoplakia?

Is oral lycopene effective in the treatment of oral leukoplakia?

\section{Oral pathology}

What are the history, clinical findings and radiographic appearance of multiple idiopathic cervical root resorption?
Having root canal obturating materials extruding rates of root canal therapy.

Stand-alone teaching improved knowledge but not skills, attitudes or behaviour. Clinically integrated teaching improved knowledge, skills, attitudes and behaviour. Teaching of EBM should be moved from classrooms to clinical practice to achieve improvements in substantial outcomes.

There was considerable variability in intent, components, construction and psychometric properties of published critical appraisal tools for research reports. There is no "gold standard" critical appraisal tool for any study design, nor is there any widely accepted generic tool that can be applied equally well across study types. No tool was specific to allied health research requirements. Thus, interpretation of critical appraisal of research reports currently needs to be considered in light of the properties and intent of the critical appraisal tool chosen for the task.

The crude annual oral cancer incidence rate attributable to leukoplakia would be between 6.2 and 29.1 cases per 100000 people.

Bidi smoking increases the risk of oral cancer. This is not only relevant for preventive efforts in South Asian countries but in Europe and the Americas where bidis are increasingly available.

Level of alcohol-drinking should form part of the risk assessment for oral cancer on an individual basis and at the population level. Advice on sensible drinking should be integral to oral cancer prevention advice in primary health care.

There is both a statistical and clinical benefit of Triclosan containing toothpaste in reduction of plaque and gingivitis.

Reductions in plaque and gingival bleeding were seen in the short term in the majority of studies reviewed. The clinical and public health significance of these changes is, however, questionable. Future studies should use longer follow-up periods to assess whether short-term beneficial changes are sustained. Other forms of oral health promotion require better quality evaluation if they are to be used to improve periodontal health.

Nonsteroidal anti-inflammatory drugs (NSAID) and cyclooxygenase (COX)-2 inhibitors had the lowest (best) numbers needed-to-treat (NNT) for the outcome of pain at least halved over 4-6h compared with placebo. These may also have fewer adverse effects after third-molar surgery, but conclusive evidence is lacking.

All leukoplakias need to be assessed histopathologically for dysplasia; Systemic beta-carotene may provide significant clinical resolution; No treatment prevents malignant transformation, histopathological worsening or recurrence; Irrespective of response to therapy, longterm follow-up is essential.

It is impossible to draw any firm conclusions from this study in the form in which it is currently reported.

Multiple idiopathic cervical root resorption was found most frequently associated with younger females. This condition appeared to be of unknown aetiology and uncertain natural history.
$100-101$ 


\section{Oral surgery}

When extraction of mandibular third molars is required, does use of a chlorhexidine ( $\mathrm{CHX}$ ) rinse reduce the incidence of dry socket (alveolar osteitis)?

Which perioperative management strategies are safe and effective for patients who are taking oral anticoagulants?

\section{Orthodontics}

Are there any long-term (minimum of 1-year postactive treatment) transverse, anteroposterior and vertical skeletal changes after rapid maxillary expansion (RME) treatment on patients with constricted arches?

Are there any long-term dental arch changes after rapid maxillary expansion (RME) treatment on patients with constricted arches?

Is early treatment of unilateral posterior crossbite effective?

Does premature birth affect oral development?

Is there an association between maternal tobacco smoking during pregnancy and nonsyndromic orofacial clefts (OFC) in infants?

What is the prevalence of dental agenesis of permanent teeth?

What are the treatment-related aetiological factors of external apical root resorption of the maxillary incisor?

\section{Paediatric dentistry}

In carious primary molars does a pulpotomy performed with ferric sulphate, compared with formocresol, result in greater clinical/radiographic success?

What are the most effective techniques for the use of conscious sedation behaviour management in paediatric dentistry?
This meta-analysis provides clinically significant evidence that repeated $\mathrm{CHX}$ mouth rinses, beginning on the day of third molar removal, reduce the incidence of dry socket but single rinses on the day of surgery do not.

Dental surgery including minor oral surgical procedures should be performed without alteration of oral anticoagulant (OAC). Heparin substituting for OAC withheld perioperatively in minor oral surgery is not supported at the present time.

Long-term transverse skeletal maxillary increase is approximately $25 \%$ of the total dental expansion for prepubertal adolescents. Better long-term outcomes are expected in transverse changes because of RME in less skeletally mature patients. RME appears not to produce clinically significant anteroposterior or vertical changes in the position of the maxilla and mandible. The conclusions from this systematic review should be considered with caution because only a secondary level of evidence was found. Long-term randomised clinical trials are needed.

The results are still inconclusive and do not really add new knowledge on the long-term effect of RME because it is based on very limited studies of second level of evidence.

The available evidence is of low quality. Alternative approaches (quad-helix, removable and fixed expansion plates) offered promising results but there are not yet data relating to their long-term stability.

This systematic review revealed contradictory results and a dearth of longitudinal studies in this subject area. Further well-designed controlled and longitudinal studies are required before any conclusions can be made regarding the consequences of premature birth on oral development.

This study showed a consistent, moderate association between maternal tobacco smoking during pregnancy and nonsyndromic OFC in infants. The effect was observed for both isolated and multiple clefts, and was stronger and more consistent for $C L \pm P$ than for $C P$.

Agenesis differs by continent and gender: the prevalence for both sexes was higher in Europe (males 4.6\% females 6.3\%) and Australia (males 5.5\%; females 7.6\%) than for North-American Caucasians (males $3.2 \%$; females $4.6 \%$ ). In addition, the prevalence of dental agenesis in females was significantly higher than in males for all three continents, with an RR of 1.37 (95\% Cl, 1.28-1.45)

Patients who require prolonged orthodontic treatment or treatment involving a significant degree of tooth movement should be warned about resorption prior to orthodontic treatment. From the available evidence, grinding of interferences appears to be the initial treatment of choice for unilateral posterior crossbites. Alternative approaches (quad-helix, removable and fixed expansion plates) offered promising results but there are not yet data relating to their long-term stability.

Ferric sulphate is as effective as formocresol for vita pulpotomies in primary teeth. 
Does the extent of discomfort differ between atraumatic restorative treatment (ART) of multisurface cavities in deciduous molars compared with use of rotary instruments?

Is there any difference in retention rate between a conventional resin sealant and a flowable restorative system used as a pit-and-fissure sealant?

\section{Periodontology}

What are the benefits and harms of routine scaling and polishing for periodontal health and do these change with different time intervals?

What is the effect of smoking and smoking cessation on the response to nonsurgical periodontal therapy in individuals who have chronic periodontitis?

Is there an association between the presence of visible third molars and periodontal pathology in a sample of middle-aged and older adults?

Does periodontal disease in pregnant mothers increase the risk of preterm birth (PTB) or low birth weight (PLBW)?

How effective is mechanical nonsurgical pocket therapy?

\section{Primary care dentistry}

What is the optimal recall frequency between dental checks?

\section{Prosthetic dentistry}

Do different occlusal schemes affect patient satisfaction in subjects who require complete dentures?

\section{Restorative dentistry}

How long do implant supported fixed partial dentures (FPDs) survive and how frequently do complications occur?

How long do conventional fixed partial dentures (FPDs) survive and how frequently do complications occur?

How long do cantilever fixed partial dentures (FPDs) survive and what effects do biological and technical complications have on them?

How long do combined tooth-implant-supported fixed partial dentures (FPDs) survive and how frequently do complications occur?

\section{Temporomandibular disorders}

Which surgical procedures, if any, can effectively treat temporomandibular articular disorders?
Children treated according to the ART approach using hand instruments alone experience less discomfort than those treated using rotary instruments.

There is some evidence to suggest that sealants using flowable restorative systems show improved retention rates, particularly for primary teeth.

There is no support for any recall regimen based on fixed intervals between scale and polishing that does not include an evaluation of clinical outcomes.

Smokers have less favourable results after nonsurgical periodontal therapy in probing depth reduction but there are no significant differences between smokers and nonsmokers in clinical attachment gain.

The presence of visible third molars may be associated with apparent periodontal pathology which could simply be increased tissue thickness related to tooth position. There is not yet any evidence of benefit from the removal of third molars in order to prevent periodontal breakdown.

There is insufficient evidence originated from observational research supporting the association between periodontal diseases and preterm birth and/or low birth weight.

Existing evidence in the form of systematic reviews provides conclusive support for the beneficial effect and efficacy of mechanical nonsurgical pocket therapy in the treatment of periodontal diseases.

Fixed recall intervals (mostly 6 months) for all patients are not evidence-based; before further evidence emerges, experienced practitioners could select low-risk individuals for extension of the recall period beyond the standard 6 months.

Despite weak evidence of improved patient satisfaction with prosthetic posterior cusped teeth, dentists are advised to continue providing patients with complete dentures with cusped posterior teeth rather than using cuspless teeth.

The survival rate of FPDs supported by implants was $95 \%$ after 5 years and $86.7 \%$ after 10 years. Although survival rate for implant supported FPDs is high, biological and technical complications are relatively common.

The 10-year probability of survival for fixed partial dentures is $89.1 \%(95 \% \mathrm{Cl} 81-93.8)$; technical complications such as loss of retention, resulted in a greater risk of FPD loss than did biological complications.

The estimated 10-year survival rate for cantilevered FPDs of $81.5 \%$, and a $63 \%$ success rate free of all complications is encouraging. However, an estimated $32.6 \%$ of abutment teeth lose vitality and over $9 \%$ become carious at 10 years, a significant finding for informed consent and professional surveillance.

Survival rates of both implants and reconstructions in combined tooth-implant-supported FPDs were lower than those reported for solely implant-supported FPDs. Hence, planning of prosthetic rehabilitation may preferentially include solely implant-supported FPDs. However, anatomical aspects, patient centred issues and risk assessments of the residual dentition may still justify combined tooth-implant-supported reconstructions.

Surgical treatments appear to have some efficacy for people who have temporomandibular articular disorders that do not respond to nonsurgical therapies.
$1 B$

$1 B$ 